\title{
«Work and Care» - Vereinbarkeit von Beruf, Privatleben und Pflege von Angehörigen
}

Ursula Steiner-König

Delegierte der FMH ins Zentralkomitee

«Tag der Kranken»
Korrespondenz:

Dr. med. Ursula Steiner-König Beim Goldenen Löwen 3

CH-4052 Basel

u.steiner[at]hin.ch
Die Zeiten, in denen Betreuung während der Berufstätigkeit einzig und allein auf Kinder zu beziehen war, sind endgültig vorbei. Wir werden älter und älter, und der Zeitpunkt kann kommen, an dem wir nicht mehr alles allein bewältigen können. Aber wer wird uns zu Hilfe kommen? Das Zentralkomitee des «Tag der Kranken» hat bewusst dieses Thema gewählt, um möglichst breit zu sensibilisieren. Probleme, die sich zunehmend auftun, gehen uns alle an.

Die traditionelle Vorstellung sähe vor, dass die Kinder der Betroffenen solche Aufgaben übernehmen. Allerdings hat sich auch da so manches verändert, Traditionen allein vermögen keine Not zu ich an Rücksicht von ihnen erwarten? Wo müsste ich meine Erwartungen anpassen? Wie reagiert mein bisheriges Umfeld auf die Tatsache, dass meine Kräfte, Zuwendung und Zeit noch von anderen Menschen beansprucht werden? Wie lange wird diese neue Mehrbelastung dauern? Welche Entlastungs- und Ablösemöglichkeiten gibt es? Müsste ich meine beruflichen Ziele wegen dieser neuen Aufgabe zurückstellen, revidieren, aufschieben? Geht das überhaupt? Was wird aus Frei- und Ferienzeit? Was ist bezahlbar und was nicht? Was wird aus unserem Budget? Wem kann ich meine Nöte, meine Verzweiflung anvertrauen? ... Der Fragekatalog ist gewiss noch lange nicht zu Ende.

\section{Die Vereinbarkeit von Beruf, Privatleben und der Pflege von}

\section{Angehörigen ist keine Privatsache, sondern eine gesamtgesellschaftliche Notwendigkeit.}

wenden. Die Erwartung, «als Eltern haben wir für euch Kinder gesorgt, nun ist es an der Zeit, dass ihr euch um uns kümmert», darf nicht mehr als Selbstverständlichkeit vorausgesetzt werden, da gerade für Frauen die berufliche Laufbahn in den letzten Jahrzehnten immer mehr an Bedeutung gewonnen hat. Hinzu kann kommen, dass neben schulpflichtigen Kindern auch noch hilfsbedürftige Eltern den Anspruch auf Zuwendung an die berufstätige Generation stellen. (Frauen werden in der Regel später Mütter als früher.) Sprach man also lange von der Doppelbelastung der Frau als Berufstätige und Hausfrau/ Mutter, steht diese heute vor einer dreifachen Aufgabe: Die Sorge um das Wohl eines oder beider Elternteile kommt noch hinzu.

Vielfältige Fragestellungen drängen sich auf: Wohnen die Eltern in der Nähe oder weit entfernt? Wer von den Geschwistern ist in der Nähe? Gibt es überhaupt Geschwister, von denen mehr erwartet werden kann, als man selbst zu leisten vermag? Falls Geschwister (oder andere nahe Verwandte) einbezogen werden können: Wie kann man sich die Aufgaben teilen? Wie gross wird der zeitliche Aufwand dessen, was geleistet werden sollte? Bin ich emotional bereit, die erforderlichen Hilfeleistungen dem Elternteil/den Eltern zukommen zu lassen? Was darf
Die oben aufgelisteten Problem- und Konfliktfelder sollen in diesem Jahr bewusst ins Zentrum des «Tag der Kranken» gestellt werden. Zwar hat das Jahr 2011 als «Jahr der Freiwilligenarbeit» manches in den Medien thematisiert, aber gerade die konfliktreichen Aspekte lassen sich nicht so einfach abhaken. Auch vonseiten der Ärzteschaft nicht. Einerseits dürfte es in unserem Praxisalltag hilfreich sein, sie im Hinterkopf zu haben ohne gleichzeitige Verpflichtung, überall helfen zu sollen (ein Allgemeininternist äusserte in einer Balintgruppe: «Ich kann doch nicht auch noch Sozialarbeiter sein!») - Andererseits geht der Appell an alle Arbeitgebenden und/ oder Vorgesetzten: Sie reagieren vermutlich häufig in Unkenntnis der Situation von Arbeitnehmenden, wenn diese beispielsweise häufiger fehlen, in ihren Leistungen nachlassen, Anzeichen eines Burn-outs geltend machen und eine Reduktion ihres Arbeitspensums wünschen. Daraus resultieren womöglich schlechtere Qualifikationen, sogar Rückstufungen oder gar Entlassungen: lauter ungerechtfertigte Resultate für die Betroffenen, hatten sie doch versucht, ihre schwierige Situation zu verschweigen und trotzdem ihre Leistung zu erbringen.

Damit gangbare Lösungen für die Zukunft entwickelt werden können, braucht es partnerschaft- 


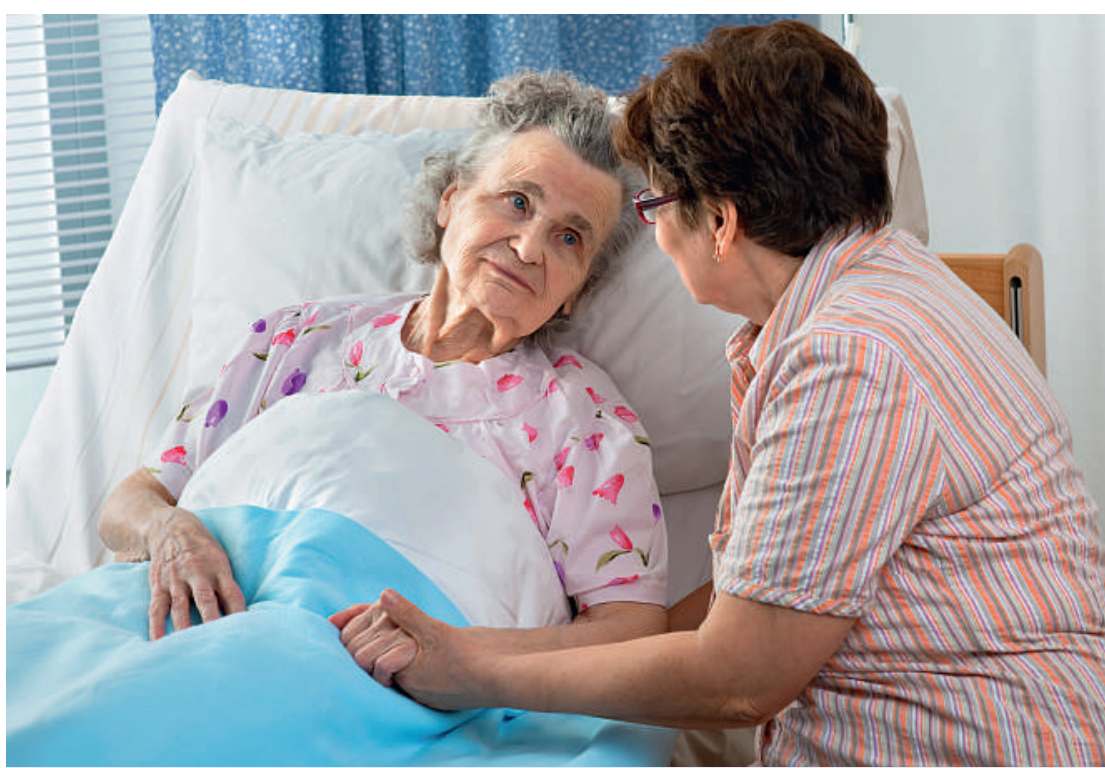

Vor allem Frauen übernehmen die Pflege kranker Angehöriger, nicht selten zusätzlich zu Beruf, Haushalt und Kindererziehung. zumeist aus osteuropäischen Ländern, die immer wieder periodisch und im Wechsel mit anderen in den gleichen Haushalt mit Betreuungsleistungen zurückkehren und von Agenturen vermittelt werden (NZZ vom 4. Januar 2012). Für die Menschen, deren Betreuung garantiert werden muss, aber oft auch für die Angehörigen, ist eine solche Lösung unbefriedigend und zusätzlich belastend. Unsere Gesundheitspolitik möchte zwar die (hoch-)betagten Menschen möglichst lange zu Hause leben lassen, um nicht noch mehr teure Pflegeplätze zur Verfügung stellen zu müssen. Was solch schmalspurige Sichtweisen alles übersehen, bleibt leider viel zu häufig verborgen und unberücksichtigt. Es wäre allerdings äusserst kurzsichtig, weiterhin darüber hinwegschauen zu wollen. Es braucht Anstrengungen von verschiedensten Seiten, um schliesslich $\mathrm{zu}$ - aus vernetzten und partnerschaftlichen Bestrebungen heraus entstandenen - Entwürfen zu gelangen, die auch politisch eine Chance auf Umsetzung haben.

Es wird viel - zu viel? - von Lebensqualität gesprochen und geschrieben. Meistens ist diejenige eines einzelnen Menschen oder eines bestimmten Kollektivs gemeint. Wenn aber nur die Lebensqualität der Patienten und Patientinnen anvisiert würde und das ist ja die von der Ärzteschaft angestrebte und vom Publikum erwartete Optik - werden Lebensqualitäten anderer in der Umgebung der Kranken unter den Teppich gekehrt. Damit würden wir den Kopf in den Sand stecken und bekämen letztlich schlechte Noten. Zum Glück müssen wir uns hier nicht allein einsetzen: Das gilt nicht nur für Ärztinnen und Ärzte, sondern genauso für alle in der Gesundheits- und Sozialpolitik engagierten Menschen. Es tut sich hier ein weiter Bereich auf, in dem nicht eine rein betriebswirtschaftliche Betrachtungsweise zum Ziel führen kann. Die Diskussion muss in der Bevölkerung breit geführt werden. Einsame politische Entscheide wären allzu praxisfern. Zusammenfassend liesse sich sagen: Menschlichkeit als gesellschaftlicher Auftrag bedarf bezüglich Umsetzung auch der Politik, und Politik bedarf bei ihrer Planung und Gesetzgebung auch der Menschlichkeit.

Zum Schluss sei verwiesen auf die Homepage des «Tag der Kranken»: www.tagderkranken.ch, wo Sie weitere Dokumente zum diesjährigen Thema finden.

\section{was in der Regel weniger tagtagliche Zusatzbe} tung, also auch leichter mit der eigenen Erwerbstätigkeit vereinbare Beanspruchung, bedeutet.

Es geht also um gesamtgesellschaftlich höchst bedeutungsvolle Problemstellungen. Abgesehen von den institutionalisierten Spitex- und weiteren Hauspflegediensten werden Lösungsansätze wie Anstellung von «Pendelmigrantinnen» praktiziert (Frauen,

\section{Literatur}

1 Bischofberger I, Höglinger M. Herausforderungen für die Vereinbarkeit von Erwerbstätigkeit und Angehörigenpflege. Schweizer Arbeitgeber. 2008; 20:36-9. 